\title{
HOMOTOPY THEORY OF MODULES OVER DIAGRAMS OF RINGS
}

\author{
J. P. C. GREENLEES AND B. SHIPLEY
}

(Communicated by Michael A. Mandell)

\begin{abstract}
Given a diagram of rings, one may consider the category of modules over them. We are interested in the homotopy theory of categories of this type: given a suitable diagram of model categories $\mathcal{M}(s)$ (as $s$ runs through the diagram), we consider the category of diagrams where the object $X(s)$ at $s$ comes from $\mathcal{M}(s)$. We develop model structures on such categories of diagrams and Quillen adjunctions that relate categories based on different diagram shapes.

Under certain conditions, cellularizations (or right Bousfield localizations) of these adjunctions induce Quillen equivalences. As an application we show that a cellularization of a category of modules over a diagram of ring spectra (or differential graded rings) is Quillen equivalent to modules over the associated inverse limit of the rings. Another application of the general machinery here is given in work by the authors on algebraic models of rational equivariant spectra. Some of this material originally appeared in the preprint "An algebraic model for rational torus-equivariant stable homotopy theory", arXiv:1101.2511, but has been generalized here.
\end{abstract}

\section{INTRODUCTION}

Given a diagram of rings, one may consider the category of modules over them. We are interested in the homotopy theory of categories of this type: given a suitable diagram of model categories $\mathcal{M}(s)$ where functors relating them are left Quillen functors, we consider the category of diagrams where the object $X(s)$ at $s$ comes from $\mathcal{M}(s)$. The purpose of this paper is to show that under suitable hypotheses, there are diagram-projective and diagram-injective model structures on the category (Theorem 3.1), and to investigate Quillen adjunctions associated to restricting the diagram (Theorems 5.3 and 5.5).

1.A. Motivation. This paper grew out of our project on algebraic models for rational $G$-equivariant spectra for $G$ a torus [8]. The main result of that project is to show that the homotopy theory of rational $G$-spectra is modelled by an algebraic category of diagrams, and it is worth describing the strategy to illustrate the use

Received by the editors September 26, 2013 and, in revised form, November 10, 2013 and March 20, 2014.

2010 Mathematics Subject Classification. Primary 55U35, 55P60, 55P42, 55P91.

The first author is grateful for support under EPSRC grant No. EP/H040692/1.

This material is based upon work by the second author supported by the National Science Foundation under grant No. DMS-1104396. 
of the techniques developed in the present paper. We begin by showing that the category of $G$-spectra is modelled by a diagram of modules over equivariant ring spectra and in the end we show that this is modelled by a category of diagrams of differential graded modules over graded rings. Some of the necessary generality is slightly hidden here, since in the spectral part we must consider a context where not only the ring, but also the group of equivariance varies with the position in the diagram. With this generality, which motivates the setting of this current paper, we are able to describe the various models we use.

The next issue is that the shape of the diagram of equivariant spectra we start with is different from the shape of the diagram of differential graded rings we end with. To relate categories based on these two diagram shapes we construct a larger diagram shape category which contains the smaller ones, so that a diagram based on the new larger shape restricts to two smaller diagrams of the original shapes. We then show that a suitable inclusion of diagram shapes induces a Quillen adjunction, and apply the Cellularization Principle [10] to show that it induces a Quillen equivalence after cellularization.

Since the techniques of using diagrams of categories and of changing the diagram shapes can be generalized and applied in other settings, we have decided to present it here in appropriate generality and we refer to [9] for the original application.

1.B. Organization. In Sections 2 and 3 , we develop model structures for categories of direct or inverse diagrams where the model category from which the objects come varies with the position in the diagram. In Section 4, as an example, we consider diagrams of modules over a diagram of ring spectra (or differential graded rings). A particularly well-known example is (differential graded) modules over the classical Hasse square which considers the integers as the pull-back ring of the rationals, the $p$-adic integers for all primes $p$, and their tensor products. Using the Cellularization Principle [10] (see also Appendix A), we show that modules over the homotopy inverse limit of a given diagram of rings can be modelled by the cellularization of the category of modules over the diagram of rings (Proposition 4.1). This is the model category version of the local to global principle for the Hasse square.

In Section 5, we consider changing diagram shapes. In particular, we consider the inclusion of a subcategory $i: \mathbf{D} \rightarrow \mathbf{E}$ and its induced restriction functor on diagram categories over $\mathbf{D}$ and $\mathbf{E}$. We then show that after certain cellularizations (or colocalizations) these different shaped diagram categories model the same homotopy theory. At the end of Section 5, we return to the inverse limit example of Section 4 to show that it is an example of this general machinery for changing diagram shapes.

1.C. Relation with other work. Diagrams of general model categories were considered in [13] (under the name Quillen presheaves) and 16], but neither of these has been published. See also Remark 3.2, See also [7] for a generalization involving the weaker notion of diagrams of Cartan-Eilenberg categories. Quillen presheaves of combinatorial model categories were considered in [3]. Since our motivation is coming from $G$-equivariant spectra for $G$ a compact Lie group, the usual models are not combinatorial. None of the references [13, [16] or 3] consider changing diagram shapes, which is crucial in our application. 


\section{Diagrams of Rings AND MOdules}

Categories of modules over diagrams of rings have created useful new models; see for example [8, 18. These examples use two underlying contexts: differential graded modules over differential graded algebras (DGAs) and module spectra over ring spectra. In [8], we needed to generalize this setting further to work with equivariant spectra.

2.A. The archetype. Given a diagram shape $\mathbf{D}$, consider a diagram of rings $R$ : $\mathbf{D} \longrightarrow \mathbb{C}$ in a symmetric monoidal category $\mathbb{C}$. Each map $R(a): R(s) \longrightarrow R(t)$ gives rise to an extension of scalars functor

$$
R(s) \text {-mod } \stackrel{a_{*}}{\longrightarrow} R(t) \text {-mod }
$$

defined by $a_{*}(X)=R(t) \bigotimes_{R(s)} X$, with right adjoint the restriction of scalars functor

$$
R(s) \text {-mod } \stackrel{a^{*}}{\longleftarrow} R(t) \text {-mod. }
$$

Now consider a category of $R$-modules; the objects are diagrams $X: \mathbf{D} \longrightarrow \mathbb{C}$ for which $X(s)$ is an $R(s)$-module for each object $s$, and for every morphism $a: s \longrightarrow t$ in $\mathbf{D}$, the map $X(a): X(s) \longrightarrow X(t)$ is a module map over the ring map $R(a)$ : $R(s) \longrightarrow R(t)$. More precisely, there is a map $X(s) \longrightarrow a^{*} X(t)$ of $R(s)$-modules (the restriction model) or, equivalently, there is a map

$$
R(t) \bigotimes_{R(s)} X(s)=a_{*} X(s) \longrightarrow X(t)
$$

of modules over the ring $R(t)$ (the extension of scalars model). Although restriction of scalars has some advantages, in the more general case, it is more natural to view the left adjoint $a_{*}$ as the primary one on the principle that the left Quillen functor dictates the direction of a Quillen pair.

2.B. A generalization. We next consider a generalization of this archetype. Here we begin with a diagram $\mathcal{M}: \mathbf{D} \longrightarrow$ Cat of model categories. These are called Quillen presheaves in [13] and in [3, 2.21] and adjunction bundles of model categories in [16]. The previous special case is $\mathcal{M}(s)=R(s)$-mod and in our applications each category $\mathcal{M}(s)$ is a category of modules in some category $\mathbb{C}(s)$ which also varies with $s$. Since $\mathcal{M}$ is a functor, for each $a: s \longrightarrow t$ in $\mathbf{D}$ we have an associated functor $a_{*}: \mathcal{M}(s) \longrightarrow \mathcal{M}(t)$ which is compatible with composition in $\mathbf{D}$. We then consider the category of $\mathcal{M}$-diagrams, $\mathcal{M}$-mod. The objects in this category consist of an object $X(s)$ from $\mathcal{M}(s)$ for each object $s$ of $\mathbf{D}$ with a transitive system of morphisms

$$
\widetilde{X}(a): a_{*} X(s) \longrightarrow X(t)
$$

for each morphism $a: s \longrightarrow t$ in $\mathbf{D}$ (the left adjoint form). If each $a_{*}$ has a right adjoint $a^{*}$, then the system of morphisms is equivalent to an adjoint system of morphisms

$$
\hat{X}(a): X(s) \longrightarrow a^{*} X(t)
$$

for each morphism $a: s \longrightarrow t$ in $\mathbf{D}$ (the right adjoint form). In [13] and [3] these objects are called sections and in [16] they are called twisted diagrams. 
2.C. Model structures. We say that $\mathcal{M}$ is a diagram of model categories if each category $\mathcal{M}(s)$ has a model structure, the functors $a_{*}$ all have right adjoints and the adjoint pair $a_{*} \vdash a^{*}$ of functors relating the model categories form a Quillen pair. For instance, the motivating example of a diagram of ring spectra (or DGAs) gives a diagram of model categories if we use the projective model structure on the category $\mathcal{M}(s)$ of $R(s)$-modules.

When $\mathcal{M}$ is a diagram of model categories, there are two ways to attempt to put a model structure on the category of $\mathcal{M}$-diagrams $\{X(s)\}_{s \in \mathbf{D}}$. The diagramprojective model structure (if it exists) has its fibrations and weak equivalences defined objectwise. The diagram-injective model structure (if it exists) has its cofibrations and weak equivalences defined objectwise. It must be checked in each particular case whether or not these specifications determine a model structure. When both model structures exist, it is clear that the identity functors define a Quillen equivalence between them.

We will prove Theorem 3.1 stating that the diagram-projective and diagraminjective model structures exist for certain diagram shapes $\mathbf{D}$.

2.D. Simple change of diagrams. Returning to the archetype diagram of modules over a diagram of rings, sometimes if we specify the modules on just part of the diagram we can fill in the remaining entries using adjoints. There are two types of examples: (1) the diagram is filled in by using left adjoints such as extension of scalars and direct limits and (2) the diagram is filled in using right adjoints such as restriction and inverse limits. In both cases, this sometimes induces a Quillen equivalence between cellularizations of categories of modules over the larger and smaller diagrams. In Section 4, we develop an example of type (2) of a Quillen equivalence of module categories. In Section [5, we develop general statements for both types (1) and (2) in the setting of diagrams of module categories as in Section 2.B.

Example 2.1. The simplest example of Type (1) starts with a diagram $R=$ $\left(R_{0} \longrightarrow R_{1}\right)$ of rings. An $R$-module gives rise to an $R_{0}$-module by evaluation at the first object. An $R_{0}$-module $X_{0}$ produces the $R$-module

$$
\left(R \bigotimes_{R_{0}} X_{0}\right)=\left(R_{0} \bigotimes_{R_{0}} X_{0} \longrightarrow R_{1} \bigotimes_{R_{0}} X_{0}\right)
$$

Example 2.2. The simplest non-trivial example of Type (2) starts with a diagram

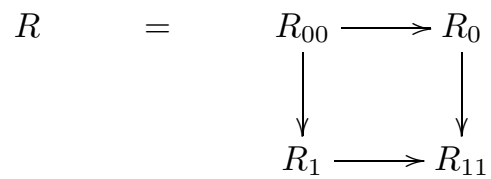

Consider $R^{\prime}=\left(R_{0} \longrightarrow R_{11} \longleftarrow R_{1}\right)$. An $R$-module gives rise to an $R^{\prime}$-module by restriction. Let $\hat{R}$ be the pullback of $R_{0} \longrightarrow R_{11} \longleftarrow R_{1}$. Let $f: R_{00} \rightarrow \hat{R}$ be the associated map. An $R^{\prime}$-module $M=\left(M_{0} \longrightarrow M_{11} \longleftarrow M_{1}\right)$ gives rise to an $\hat{R}$-module $\hat{M}$ by pullback. This in turn gives rise to an $R$-module which agrees with the original $R^{\prime}$-module $M$, except over $R_{00}$ the induced module is $f^{*} \hat{M}$. 
Returning to the general case in more detail, we let $i: \mathbf{D} \longrightarrow \mathbf{E}$ be the inclusion of a full subcategory, and $R: \mathbf{E} \longrightarrow \mathbb{C}$ be a diagram of rings. We restrict $R$ to a diagram $\left.R\right|_{\mathbf{D}}: \mathbf{D} \longrightarrow \mathbb{C}$, and this induces a restriction functor

$$
i^{*}: R-\left.\bmod \longrightarrow R\right|_{\mathbf{D}^{-} \text {mod }}
$$

We discuss two cases in detail in Section 5, depending on whether we focus on $i^{*}$ as a right or left adjoint.

The left adjoint case. If $i^{*}$ has a left adjoint $i_{*}$ and we consider diagram-projective model structures (with objectwise weak equivalences and fibrations) on the two categories, then the adjunction $\left(i_{*}, i^{*}\right)$ is a Quillen pair.

In fact for a diagram $M$ on $\mathbf{D}$, we may identify $i_{*} M$ explicitly. To find its value at an object $t$ of $\mathbf{E}$ we consider the category $\mathbf{D} / t$ whose objects are morphisms $s \longrightarrow t$ in $\mathbf{E}$ with $s$ in $\mathbf{D}$ and then take $i_{*} M(t)=\lim _{s \in \mathbf{D} / t} a_{*} M(s)$ (closely related to the latching object at $t$ ). In particular, if objects of $\mathbf{D}$ have no automorphisms and $s$ is in $\mathbf{D}$, then $\mathrm{id}_{s}$ is a terminal object of $\mathbf{D} / s$ and $i_{*}$ will not change the value at $s$. In this case, $i_{*}$ leaves the entries in $\mathbf{D}$ unchanged and the unit $M \longrightarrow i^{*} i_{*} M$ is an isomorphism.

The right adjoint case. Similarly, if $i^{*}$ has a right adjoint, $i_{!}$, and we consider diagram-injective model structures (with objectwise weak equivalences and cofibrations) then the adjunction $\left(i^{*}, i_{!}\right)$is a Quillen pair.

In fact for a diagram $M$ on $\mathbf{D}$, we may identify $i_{!} M$ explicitly. To find its value at an object $t$ of $\mathbf{E}$, we consider the category $t / \mathbf{D}$ whose objects are morphisms $t \longrightarrow s$ in $\mathbf{E}$ with $s$ in $\mathbf{D}$, and then take $i_{!} M(t)=\lim _{\leftarrow s \in t / \mathbf{D}} a^{*} M(s)$ (closely related to the matching object at $t$ ). In particular, if objects of $\mathbf{D}$ have no automorphisms and $s$ is in $\mathbf{D}$, then $\mathrm{id}_{s}$ is an initial object of $s / \mathbf{D}$ and $i_{\text {! will not change the value }}$ at $s$. In this case, $i_{\text {! }}$ leaves the entries in $\mathbf{D}$ unchanged and the counit $i^{*} i_{!} M \longrightarrow M$ is an isomorphism.

\section{Diagram-injective model Structures}

In this section we develop diagram-projective and diagram-injective model structures for the generalized categories of diagrams defined in Section 2.B. This is discussed in more detail in the preprint [16, 3.2.13, 3.3.5]. As we see in Remark 3.4 below, one familiar example of such a generalized category of diagrams is the category of modules over a ring with many objects, [18, 3.3.2]. In that case, the diagram-projective (or standard) model structure here agrees with the one developed in [18, A.1.1] and has objectwise weak equivalences and fibrations; see also [1]. In contrast, the diagram-injective model structure here has weak equivalences and cofibrations determined at each object. These are the analogues of the model structures for diagrams over direct and inverse small categories developed, for example, in [14, 5.1.3].

We restrict our attention here to the diagrams indexed on small direct (or inverse) categories. Let $\mathbf{D}$ be a small direct category with a fixed linear extension $d: \mathbf{D} \rightarrow \lambda$ for some ordinal $\lambda$. Note that if $\mathbf{D}(s, t)$ is non-empty and $s \neq t$, then $d(s)<d(t)$. Let $\mathcal{M}$ be a diagram of model categories indexed by $\mathbf{D}$; that is, each $s \in \mathbf{D}$ is assigned a model category $\mathcal{M}(s)$ and each $a: s \rightarrow t$ in $\mathbf{D}$ is assigned a left Quillen functor $a_{*}: \mathcal{M}(s) \rightarrow \mathcal{M}(t)$ (with right adjoint $a^{*}$ ) which are compatible 
with composition. Then a diagram $X$ over $\mathcal{M}$ (or " $\mathcal{M}$-diagram") specifies for each object $s$ in $\mathbf{D}$ an object $X(s)$ of $\mathcal{M}(s)$ and for each morphism $a: s \rightarrow t$ in $\mathbf{D}$ a map $\tilde{X}(a): a_{*} X(s) \rightarrow X(t)$, again compatible with compositions. Let $\mathbf{D}_{t}$ be the category whose objects are all non-identity maps in $\mathbf{D}$ with codomain $t$. Then any diagram $X$ induces a functor from $\mathbf{D}_{t}$ to $\mathcal{M}(t)$ by taking $a: s \rightarrow t$ in $\mathbf{D}_{t}$ to $a_{*} X(s)$. Define the latching space functor, $L_{t} X$ as the direct limit in $\mathcal{M}(t)$,

$$
L_{t} X=\lim _{\rightarrow \mathbf{D}_{t}} a_{*} X(s) .
$$

In the dual situation where $\mathbf{D}$ is a small inverse category, we consider again a diagram of model categories $\mathcal{M}$. Note, here again each $a: s \rightarrow t$ in $\mathbf{D}$ is assigned to a left Quillen functor $a_{*}: \mathcal{M}(s) \rightarrow \mathcal{M}(t)$ with right adjoint $a^{*}$. Let $\mathbf{D}^{s}$ be the category of all non-identity maps in $\mathbf{D}$ with domain $s$. Then any $\mathcal{M}$ diagram $X$ induces a functor from $\mathbf{D}^{s}$ to $\mathcal{M}(s)$ by taking $a: s \rightarrow t$ in $\mathbf{D}^{s}$ to $a^{*} X(t)$. Define the matching space functor, $M_{i} X$ as the inverse limit in $\mathcal{M}(i)$,

$$
M_{s} X=\lim _{\leftarrow \mathbf{D}^{s}} a^{*} X(t) .
$$

Theorem 3.1. Assume we are given a category $\mathbf{D}$ and a diagram of model categories, $\mathcal{M}$, indexed on $\mathbf{D}$ as above.

(i) If $\mathbf{D}$ is a direct category, then there is a diagram-projective model structure on the category of diagrams over $\mathcal{M}$ with objectwise weak equivalences and fibrations; that is, $X \rightarrow Y$ is a weak equivalence (or fibration) if $X(s) \rightarrow Y(s)$ is an underlying weak equivalence (or fibration) in $\mathcal{M}(s)$ for all $s$. This map is a (trivial) cofibration if and only if the induced map $X(s) \coprod_{L_{s} X} L_{s} Y \rightarrow Y(s)$ is a (trivial) cofibration in $\mathcal{M}(s)$ for all $s$.

(ii) If $\mathbf{D}$ is an inverse category, then there is a diagram-injective model structure on the category of diagrams over $\mathcal{M}$ with objectwise weak equivalences and cofibrations; that is, $X \rightarrow Y$ is a weak equivalence (or cofibration) if $X(s) \rightarrow Y(s)$ is an underlying weak equivalence (or cofibration) in $\mathcal{M}(s)$ for all $s$. This map is a (trivial) fibration if and only if the induced map $X(s) \rightarrow Y(s) \times_{M_{s} Y} M_{s} X$ is a (trivial) fibration in $\mathcal{M}(s)$ for all $s$.

Proof. The verification of the axioms follows the same outline as in [14, 5.1.3]. The only difference is that here the ambient category changes at each object in D. Instead of repeating these arguments, we give some of the details for these changing categories. As in [14, 5.1.3] we consider only the direct category case, since the inverse category case is dual.

Define $\mathbf{D}_{<\beta}$ as the full subcategory of $\mathbf{D}$ on all objects $i$ such that $d(s)<\beta$. Then let $\mathcal{M}_{<\beta}$ denote the diagram of model categories induced by the restriction of $\mathcal{M}$ to $\mathbf{D}_{<\beta}$. Similarly, for any $\mathcal{M}$ diagram $X$, the restriction to $\mathbf{D}_{<\beta}$ gives an $\mathcal{M}_{<\beta}$ diagram $X_{<\beta}$. Given these definitions, the lifting axioms follow by induction as in [14, 5.1.4], by producing lifts for the various restrictions to $\mathcal{M}_{<\beta}$ diagrams. Note that at the successor ordinal case the relevant commutative diagram is just a usual lifting problem in $\mathcal{M}(s)$.

To complete the verification of the model structure we follow the proof of 14 , 5.1.3]. That proof uses [14, 5.1.5] to consider maps formed by colimits. In the usual setting, the colimit is the left adjoint to the constant functor. Here though instead of the constant functor one must use the relevant right adjoint. The colimit 
in question in our analogue of the proof of [14,5.1.3] is the functor $L_{s}$; denote its right adjoint by $G_{s}$. For an object $X$ in $\mathcal{M}(s)$, the $\mathcal{M}$ diagram $G_{s} X$ at $a \in \mathbf{D}_{s}$ is $a^{*} X$. Since each $a^{*}$ is a right Quillen functor, $G_{s}$ takes (trivial) fibrations to objectwise (trivial) fibrations. Thus the required analogue of [14, 5.1.5] holds in our setting as well.

The only other change needed in the proof of [14,5.1.3] is that for the induction step in the construction of the functorial factorizations one uses factorization in $\mathcal{M}(s)$ to factor the map $X(s) \coprod_{L_{s} X} L_{s} Y \rightarrow Y(s)$.

Remark 3.2. As mentioned in Section 1.C the proof of this theorem is given in much more detail in [16, 3.2.13, 3.3.5]. In [13, 17.1] Reedy diagrams are considered; see also [16, 3.3.6] and [17. In [16, 3.4.5], the projective model structure for general diagram shapes is considered for cofibrantly generated model categories. Since our motivating examples are diagrams over direct (or inverse) categories, we do not consider this generality here. Projective and injective model structures for general diagram shapes are also considered in [3] for combinatorial model categories.

For the applications in 9], we only need to consider diagram categories $\mathbf{D}$ with at most one map between any two objects. Restricting to this situation simplifies the arguments for the following proposition.

Proposition 3.3. Let $\mathbf{D}$ be a direct (or inverse) category with at most one map between any two objects. Assume given a diagram of proper, cellular model categories $\mathcal{M}$; that is, for each $s \in \mathbf{D}$, the model structure $\mathcal{M}(s)$ is proper and cellular. Then the diagram-projective (or diagram-injective) model structure on $\mathcal{M}$-diagrams defined in Theorem 3.1 is a proper, cellular model category.

Proof. We first establish properness. In the diagram-projective case fibrations and weak equivalences are defined objectwise and one can show that any cofibration induces an objectwise cofibration. Since pullbacks and pushouts are constructed at each object and $\mathcal{M}(s)$ is assumed to be a proper model structure for each $s$, properness follows. The diagram-injective case is dual.

We use Hirschhorn's treatment of Reedy categories [12, Chapter 15] to establish that these model structures are cellular. Note that a direct category is an example of a Reedy category with no morphisms that lower degrees. In this case, the matching categories are empty so that the matching objects are just the terminal object. Thus, the Reedy fibrations are just the objectwise fibrations and the Reedy model structure [12, 15.3.4] agrees with the diagram-projective model structure defined above. The arguments for an inverse category are dual.

Next we define the generating (trivial) cofibrations. Given an object $A$ in $\mathcal{M}(s)$, define the free $\mathcal{M}$ diagram generated by $A$ at $s$ to be $\mathcal{F}_{A}^{s}(t)=a_{*} A$ when $\mathbf{D}(s, t)=$ $\{a\}$ is non-empty and the initial object otherwise. For $\mathbf{D}$ a direct category, and $f: A \rightarrow B$ in $\mathcal{M}(s)$, define $R F_{f}^{s}$ to be the induced map of diagrams $\mathcal{F}_{A}^{s} \rightarrow \mathcal{F}_{B}^{s}$. Let $I_{s}$ denote the generating cofibrations for $\mathcal{M}(s)$. Let $R F_{I}^{\mathbf{D}}$ denote the set of maps $R F_{f}^{s}$ for all maps $f$ in $I_{s}$ for all $s$ in $\mathbf{D}$. Define $R F_{J}^{\mathbf{D}}$ similarly based on the sets $J_{s}$ of generating trivial cofibrations for $\mathcal{M}(s)$. By [12, 15.6.27], the diagramprojective model structure on $\mathcal{M}$ diagrams is cofibrantly generated with generating cofibrations $R F_{I}^{\mathbf{D}}$ and generating trivial cofibrations $R F_{J}^{\mathbf{D}}$. 
For $\mathbf{D}$ an inverse category, we define the boundary of the free functor $\mathcal{F}_{X}^{s}$ to be $\partial \mathcal{F}_{X}^{s}(t)=a_{*} X$ when $\mathbf{D}(s, t)=\{a\}$ is non-empty and $s \neq t$ and the initial object otherwise. Note that these functors only differ at $s=t$. Given a map $f: A \rightarrow B$ in $\mathcal{M}(s)$, let $R F_{f}^{s}$ denote the $\mathcal{M}$ diagram map

$$
\mathcal{F}_{A}^{s} \coprod_{\partial \mathcal{F}_{A}^{s}} \partial \mathcal{F}_{B}^{s} \rightarrow \mathcal{F}_{B}^{s}
$$

(In [12, 15.6.18], the boundary $\partial \mathcal{F}^{s}$ of a free functor is defined for general Reedy categories and uses the non-identity maps in $\mathbf{D}$ which lower degree. For $\mathbf{D}$ a direct category, this simplifies since no map lowers degree. Thus, $\partial \mathcal{F}^{s}$ is just the diagram of initial objects and one recovers the above definition of $R F_{f}^{s}$.) As in the case of a direct category, by [12, 15.6.27], the diagram-injective model structure on $\mathcal{M}$ diagrams is cofibrantly generated with generating cofibrations $R F_{I}^{\mathbf{D}}$ and generating trivial cofibrations $R F_{J}^{\mathrm{D}}$ defined as above using the generating cofibrations $I_{s}$ and trivial cofibrations $J_{s}$ from $\mathcal{M}(s)$.

Finally, [12, 15.7.6] establishes the additional conditions for showing this is a cellular model category given that each category $\mathcal{M}(s)$ is a cellular model category.

Remark 3.4. For $\mathbf{D}$ an inverse category with at most one morphism in each $\mathbf{D}(s, t)$, modules over a diagram of rings over $\mathbb{C}$ are equivalent to categories of modules over a ring with many objects over $\mathbb{C}$. If $\mathbf{D}(s, t)$ is non-empty, then the map $R(s) \rightarrow R(t)$ makes $R(t)$ an $R(s)$-module. There is an associated $\mathbb{C}$-enriched category indexed on the objects of $\mathbf{D}$ which we also denote by $R$ with $R(s, s)$ the ring $R(s), R(s, t)$ trivial when $\mathbf{D}(s, t)$ is empty, and $R(s, t)$ the $R(t)-R(s)$ bimodule $R(t)$ when $\mathbf{D}(s, t)$ is non-empty.

A (left) module $M$ over $R$ is a covariant $\mathbb{C}$-enriched functor from $R$ to $\mathbb{C}$. The data needed to specify such a module is exactly the same as given for a module over the associated diagram $R$. First, for each object $s$ in $\mathbf{D}, M(s)$ is an $R(s, s)(=R(s))$ module and for each morphism $a: s \rightarrow t$ in $\mathbf{D}$ the module structure specifies a map $R(s, t) \bigotimes_{R(s, s)} M(s) \rightarrow M(t)$. Since $R(s, t)=R(t)$, this is the required map $F_{a} M(s) \rightarrow M(t)$ where $F_{a}$ is extension of scalars over $R(s) \rightarrow R(t)$. We consider covariant functors here because this eases the comparison with diagrams even though this differs from the right modules (or contravariant functors) considered in [18, 3.3.2].

Due to this equivalence of categories the application in the following section could also be developed in the setting of modules over a ring with many objects.

\section{INVERSE LIMIT EXAMPLE}

In this section we develop a result comparing modules over a diagram of rings and modules over the homotopy inverse limit of the diagram of rings. We show that the adjunction associated to the change from the diagram of rings to the one homotopy inverse limit ring induces a Quillen equivalence after applying the Cellularization Principle from [10; see also Proposition A.1. This is a model for the more general adjunctions considered in Section 5. The particular case of a pullback diagram of rings, such as in the classical Hasse principle, is treated in more detail in Section 6 of [10. Here we will work in the context of ring and module spectra, but this material also easily translates to the differential graded context. Note 
though that it is necessary to be in a stable context to use Proposition A.1 and here $A$-cellularization denotes cellularization with respect to all suspensions and desuspensions of $A$.

Assume we are given a finite, inverse category $\mathbf{D}$ with at most one morphism in each $\mathbf{D}(s, t)$ and a diagram of ring spectra, $R$, indexed on $\mathbf{D}$. We consider the associated diagram of model categories $\mathcal{M}$ with $\mathcal{M}(s)$ the model category of $R(s)$ module spectra and $F_{a}=R(t) \wedge_{R(s)}(-)$ the left Quillen functor given by extension of scalars. We refer to $\mathcal{M}$-diagrams as $R$-modules and compare the diagram-injective model category of $R$-modules with modules over the homotopy inverse limit of the diagram $R$.

By [12, 19.9.1], the homotopy inverse limit of $R$ is the inverse limit of a fibrant replacement of $R$ in the diagram-injective model category of $\mathbf{D}$-diagrams of ring spectra. This model structure exists by [14, 5.1.3]; see also [12, 15.3.4] since an inverse category is a particular example of a Reedy category. Let $g: R \rightarrow f R$ be this fibrant replacement and let $\hat{R}$ denote the inverse limit over $\mathbf{D}$ of $f R$. We compare $R$-modules and $\hat{R}$-modules via the category of $f R$-modules. Since $R \rightarrow$ $f R$ is an objectwise weak equivalence, there is a Quillen equivalence between $R$ modules and $f R$-modules by Lemma 4.2 below. We also establish below a Quillen adjunction between $\hat{R}$-modules and $f R$-modules which is a Quillen equivalence after cellularization. To satisfy the smallness hypotheses needed in the Cellularization Principle A.1. we must assume that $\mathbf{D}$ is a finite category. This leads to the following statement.

Proposition 4.1. For $\mathbf{D}$ a finite, inverse category with at most one morphism in each $\mathbf{D}(s, t)$ and $R$ a $\mathbf{D}$-diagram of ring spectra with homotopy inverse limit $\hat{R}$, there is a zig-zag of Quillen equivalences between the category of $\hat{R}$-modules and the cellularization with respect to $R$ of $R$-modules,

$$
\hat{R}-\bmod \simeq_{Q} R \text {-cell-R-mod. }
$$

We first need the following lemma.

Lemma 4.2. Assume we are given $L: \mathcal{M} \rightarrow \mathcal{N}$ a map of diagrams of model categories over a direct category $\mathbf{D}$. If each $L(s): \mathcal{M}(s) \rightarrow \mathcal{N}(s)$ is a left Quillen equivalence, then $L$ induces a Quillen equivalence between the diagram-projective model structures of $\mathcal{M}$ and $\mathcal{N}$ diagrams. If $\mathbf{D}$ is instead an inverse category, then $L$ induces a Quillen equivalence between the diagram-injective model structures.

Proof. Let $R$ denote the right adjoint of $L$. Since each $R(s)$ is a right Quillen functor, $R$ preserves the objectwise fibrations and weak equivalences of the diagramprojective model structures. The equivalence then follows since a cofibrant or fibrant $\mathcal{M}$ diagram is objectwise cofibrant or fibrant in the diagram-projective model structure. Namely, given a cofibrant $\mathcal{M}$ diagram $X$ and a fibrant $\mathcal{N}$ diagram $Y$, a map $L X \rightarrow Y$ is an objectwise weak equivalence if and only if $X \rightarrow R Y$ is an objectwise weak equivalence since $L$ and its right adjoint $R$ are objectwise Quillen equivalences. The diagram-injective case is similar.

Proof of Proposition 4.1. Since $g: R \rightarrow f R$ is an objectwise weak equivalence, and extension of scalars along weak equivalences of ring spectra induce Quillen equivalences, the associated diagram module categories are Quillen equivalent by Lemma 4.2. Once we verify that $R$ is small (and hence also $f R$ is small), Corollary A.2 shows that this induces a Quillen equivalence on the cellularizations of the 
diagram-injective model structures

$$
R \text {-cell- } R \text {-mod } \simeq_{Q} f R \text {-cell- } f R \text {-mod }
$$

since $R$ is cofibrant in $R$-modules and extension of scalars takes $R$ to $f R$.

To show that the object $R$ is small in $R$-modules, we first show it is the finite colimit of small objects in $R$-modules. Let $L^{s}$ denote the left adjoint to evaluation at the object $s \in \mathbf{D}$. Note that the objects $L^{s} R(s)$ are small because $R(s)$ is small in $R(s)$-modules and the right adjoint, evaluation, commutes with infinite coproducts. One can show that $L^{s} R(s)$ is the $R$-module with value $R(t)$ at $t \in \mathbf{D}$ if $\mathbf{D}(s, t)$ is non-empty and 0 otherwise. Thus, if $\mathbf{D}(s, t)$ is non-empty there is a map $L^{t} R(t) \rightarrow L^{s} R(s)$ and $R$ is the (finite) colimit over $\mathbf{D}^{o p}$ of $L^{s} R(s)$.

We use [12, 19.9.1] to show that this colimit is a homotopy colimit. This follows since $\mathbf{D}^{o p}$ is a direct category and the diagram $L^{s} R(s)$ is Reedy cofibrant because all of the maps between objects $L^{s} R(s)$ and $L^{t} R(t)$ are either the inclusion of 0 or the identity map.

To show that the finite homotopy colimit of small objects is small, we show that the adjoint finite limit commutes with infinite direct sums of abelian groups. A finite product commutes with an infinite coproduct because finite products agree with finite coproducts here. One can also check directly that equalizers commute with infinite coproducts for abelian groups. Since finite limits are constructed from finite products and equalizers, this shows that finite limits commute with infinite coproducts. This argument is worked out in more detail for $\mathbf{D}$ a pullback diagram in Section 6.4 of 10 .

Next we compare $\hat{R}$-modules and the diagram-injective structure on $f R$-modules. Since $\hat{R}$ is the inverse limit of $f R$, any $f R$-module $M$ defines an underlying $\mathbf{D}$ diagram of $\hat{R}$-modules $\widetilde{M}$. Denote the inverse limit of $\widetilde{M}$ by $\hat{M}$. The functor $f R \otimes_{\hat{R}}$ - is left adjoint to this inverse limit functor and takes an $\hat{R}$ module $N$ to the $f R$-module with $f R(s) \bigotimes_{\hat{R}} N$ at $s \in \mathbf{D}$. Since extension of scalars for a map of ring spectra is a left Quillen functor and cofibrations and weak equivalences are defined objectwise, $f R \bigotimes_{\hat{R}}-$ is a left Quillen functor from $\hat{R}$-modules to $f R$-modules. We next apply the Cellularization Principle, Proposition A.1 (2), to this Quillen adjunction to induce a Quillen equivalence on the appropriate cellularizations. Note that $\hat{R}$ is cofibrant as an $\hat{R}$-module and applying extension of scalars to it gives $f R$. Since $f R$ is diagram-injective fibrant as a diagram of ring spectra, it is also diagram-injective fibrant as an $f R$-module. Since $\hat{R}$ is the inverse limit of $f R$, cellularization induces a Quillen equivalence,

$$
\hat{R} \text {-cell- } \hat{R} \text {-mod } \simeq_{Q} f R \text {-cell- } f R \text {-mod. }
$$

Since $\hat{R}$ is already a cofibrant generator of $\hat{R}$-modules, the cellular weak equivalences and fibrations in $\hat{R}$-cell- $\hat{R}$-modules agree with those before cellularization. Thus the cellularization of the model structure on the left is unnecessary and the statement follows.

Remark 4.3. We want to point out that the model category $R$-cell- $R$-mod is similar to the homotopy limit homotopy theory considered in [19], [3], [5], and [6]. However, in the present case we have shown this is a model for the simpler category of $\hat{R}$ modules.

Also see Section 5.C for a reconsideration of the results here using the general results of Section 5 . 


\section{Adjunctions}

In this section we develop Quillen equivalences between categories of modules over diagrams of different shapes. We consider the two basic cases corresponding to left and right adjoints as described in Section 2.D. We end by showing how these two base cases were combined in Proposition 4.1.

5.A. The left adjoint case. Suppose $\mathbf{E}$ is a direct category with a fixed linear extension $d: \mathbf{E} \rightarrow \lambda$ for some ordinal $\lambda$. Let $i: \mathbf{D} \longrightarrow \mathbf{E}$ be an inclusion of a full subcategory and $\mathcal{M}: \mathbf{E} \longrightarrow \mathbb{C}$ be a diagram of model categories and Quillen adjunctions as in Sections 2.B and 2.C. Restriction of $\mathcal{M}$ produces a diagram $\left.\mathcal{M}\right|_{\mathbf{D}}: \mathbf{D} \longrightarrow \mathbb{C}$, and a restriction functor from $\mathcal{M}$-diagrams to $\left.\mathcal{M}\right|_{\mathbf{D}}$-diagrams

$$
i^{*}: \mathcal{M}-\left.\bmod \longrightarrow \mathcal{M}\right|_{\mathbf{D}^{-} \text {mod }}
$$

Assuming that each model category $\mathcal{M}(s)$ has all colimits, a left adjoint, $i_{*}$, of this

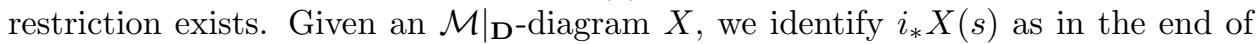
Section 2.D. Let $\mathbf{D} / t$ be the category of morphisms $a: s \rightarrow t$ in $\mathbf{E}$ with domain $s$ in D. Then

$$
i_{*} X(t)=\lim _{s \in \mathbf{D} / t} a_{*} X(s) .
$$

Since $\mathbf{D}$ is a full subcategory, for any $a^{\prime}: t \rightarrow t^{\prime}$ in $\mathbf{E}$ the structure maps $a_{*}^{\prime} i_{*} X(t) \rightarrow$ $i_{*} X\left(t^{\prime}\right)$ can be filled in by the universal property of colimits and the compatibility of compositions of arrows in $\mathbf{E}$.

Example 5.1. Consider a category $\mathbf{E}$ with three objects and three non-identity morphisms

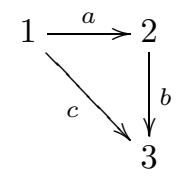

with $b \circ a=c$ and let $\mathbf{D}$ be the full subcategory $1 \stackrel{c}{\rightarrow} 3$. Assume we are given a diagram of rings $R$ over $\mathbf{E}$ and an $\left.R\right|_{\mathbf{D}^{-m o d u l e}} M$ with structure map

$$
c_{*} M(1)=R(3) \bigotimes_{R(1)} M(1) \rightarrow M(3) .
$$

Then $i_{*} M(2)=a_{*} M(1)=R(2) \bigotimes_{R(1)} M(1)$ and the structure map

$$
b_{*} i_{*} M(2)=b_{*} a_{*} M(1) \rightarrow M(3)
$$

agrees with the structure map $c_{*} M(1) \rightarrow M(3)$.

In our applications for 9, the inclusion of $\mathbf{D}$ in $\mathbf{E}$ is similar to this example and thus has a simplified left adjoint. This situation is described in the following statement.

Proposition 5.2. Let $i: \mathbf{D} \longrightarrow \mathbf{E}$ be the inclusion of a full subcategory $\mathbf{D}$ in a direct category $\mathbf{E}$ such that $\mathbf{D} / s$ has a terminal object $a: t_{s} \rightarrow s$ for each $s \in \mathbf{E}$. Given an $\left.\mathcal{M}\right|_{\mathbf{D}}$-diagram $X$, the left adjoint to restriction evaluated at $s$ is $i_{*} X(s)=$ $a_{*} X\left(t_{s}\right)$. 


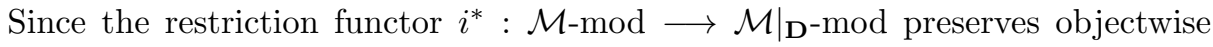
weak equivalences and fibrations, it is a right Quillen functor on the diagramprojective model structures. It then induces a Quillen equivalence on the cellularizations under the following conditions by the Cellularization Principle A.1.

Theorem 5.3. Let $\mathbf{E}$ be a direct category with $i: \mathbf{D} \longrightarrow \mathbf{E}$ an inclusion of a full subcategory and let $\mathcal{M}: \mathbf{E} \longrightarrow \mathbb{C}$ be a diagram of right proper, cellular, stable model categories such that each $\mathcal{M}(s)$ has all colimits. There is a Quillen adjunction on the diagram-projective model structures,

$$
i_{*}:\left.\mathcal{M}\right|_{\mathbf{D}}-\bmod \longrightarrow \mathcal{M}-\bmod : i^{*} .
$$

(1) Assume we are given a stable set of small cells $\mathcal{X}$ in $\left.\mathcal{M}\right|_{\mathbf{D}}$-mod such that the derived counit $X \rightarrow \underline{i}^{*} \underline{i}_{*} X$ is an equivalence for each $X \in \mathcal{X}$. Then $\left(i_{*}, i^{*}\right)$ induces a Quillen equivalence on the associated cellularizations,

$$
\mathcal{X}-\text { cell- }\left.\mathcal{M}\right|_{\mathbf{D}}-\bmod \simeq_{Q} \underline{i}_{*} \mathcal{X}-\text { cell-M-mod. }
$$

(2) Assume we are given a stable set of small cells $\mathcal{Y}$ in $\mathcal{M}$-mod such that $\underline{i}^{*} Y$ is small and the derived counit $\underline{i}_{*} \underline{i}^{*} Y \rightarrow Y$ is an equivalence for each $Y \in \mathcal{Y}$. Then $\left(i_{*}, i^{*}\right)$ induces a Quillen equivalence on the associated cellularizations,

$$
\underline{i}^{*} \mathcal{Y}-\text { cell- }\left.\mathcal{M}\right|_{\mathbf{D}}-\bmod \simeq_{Q} \mathcal{Y}-\text { cell-M-mod }
$$

Remark 5.4. In (1) above we do not need the hypothesis that $\underline{i}_{*} X$ is small in $\mathcal{M}$-mod, because it follows from $X$ being small in $\left.\mathcal{M}\right|_{\mathbf{D}^{-}}$mod and the fact that $i^{*}$ preserves all weak equivalences and commutes with colimits. When $\mathbf{D}$ is finite there is a corresponding simplification in (2), since if $Y$ is small in $\mathcal{M}$-mod, then it is automatic that $\underline{i}^{*} Y$ is small. Here we use that finite limits agree with finite colimits in stable model categories and hence commute with filtered colimits.

5.B. The right adjoint case. The right adjoint case is dual to the left adjoint case above; we spell out some of the details here. Let $\mathbf{E}$ be an inverse category with a fixed linear extension $d: \mathbf{E}^{o p} \rightarrow \lambda$ for some ordinal $\lambda$. Let $i: \mathbf{D} \longrightarrow \mathbf{E}$ be an inclusion of a full subcategory and $\mathcal{M}: \mathbf{E} \longrightarrow \mathbb{C}$ be a diagram of model categories and Quillen adjunctions as in Sections 2.B and 2.C Restriction of $\mathcal{M}$ produces a diagram $\left.\mathcal{M}\right|_{\mathbf{D}}: \mathbf{D} \longrightarrow \mathbb{C}$, and a restriction functor from $\mathcal{M}$-diagrams to $\left.\mathcal{M}\right|_{\mathbf{D}}$-diagrams

$$
i^{*}: \mathcal{M}-\left.\bmod \longrightarrow \mathcal{M}\right|_{\mathbf{D}} \text { mod. }
$$

Assuming that each model category $\mathcal{M}(i)$ has all limits, a right adjoint, $i_{\text {! }}$, of this restriction exists. Given an $\left.\mathcal{M}\right|_{\mathbf{D}^{-}}$diagram $X$, we identify $i_{!} X(t)$ as in the end of Section 2.D. Let $t / \mathbf{D}$ be the category of morphisms $a: t \rightarrow s$ in $\mathbf{E}$ with codomain $s$ in $\mathbf{D}$. Then

$$
i_{!} X(t)=\lim _{\leftarrow \in t / \mathbf{D}} a^{*} X(s) .
$$

Note that for $a^{\prime}: t^{\prime} \rightarrow t$ in $\mathbf{E}$ the structure maps $i_{!} X\left(t^{\prime}\right) \rightarrow a^{\prime *} i_{!} X(t)$ can be filled in by the universal property of limits and the compatibility of compositions of arrows in $\mathbf{E}$ since $\mathbf{D}$ is a full subcategory.

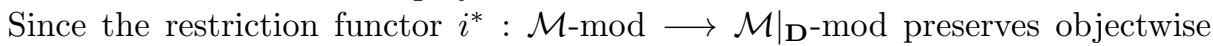
weak equivalences and cofibrations, it is a left Quillen functor on the diagraminjective model structures. It then induces a Quillen equivalence on the cellularizations under the following conditions by the Cellularization Principle A.1. For (1), see also Remark 5.4. 
Theorem 5.5. Let $\mathbf{E}$ be an inverse category with $i: \mathbf{D} \longrightarrow \mathbf{E}$ an inclusion of a full subcategory and let $\mathcal{M}: \mathbf{E} \longrightarrow \mathbb{C}$ be a diagram of right proper, cellular, stable model categories such that each $\mathcal{M}(s)$ has all limits. There is a Quillen adjunction on the diagram-injective model structures,

$$
i^{*}: \mathcal{M}-\left.\bmod \longrightarrow \mathcal{M}\right|_{\mathbf{D}}-\bmod : i_{!} .
$$

(1) Assume we are given a stable set of small cells $\mathcal{X}$ in $\mathcal{M}$-mod such that $\underline{i}^{*} X$ is small and the derived counit $X \rightarrow \underline{i}_{1} \underline{i}^{*} X$ is an equivalence for each $X \in \mathcal{X}$. Then $\left(i^{*}, i_{1}\right)$ induces a Quillen equivalence on the associated cellularizations,

$$
\mathcal{X} \text {-cell-M-mod } \simeq_{Q} \underline{i}^{*} \mathcal{X} \text {-cell-M }\left.\mathcal{M}\right|_{\mathbf{D}}-\bmod .
$$

(2) Assume we are given a stable set of small cells $\mathcal{Y}$ in $\left.\mathcal{M}\right|_{\mathbf{D}}$-mod such that $\underline{i}_{1} Y$ is small and the derived counit $\underline{i}^{*} \underline{i}_{!} Y \rightarrow Y$ is an equivalence for each $Y \in \mathcal{Y}$. Then $\left(i^{*}, i_{1}\right)$ induces a Quillen equivalence on the associated cellularizations,

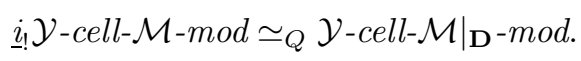

5.C. Inverse limit example revisited. In Proposition 4.1 a zig-zag of Quillen equivalences was used to produce a model for the category of modules over an inverse limit ring as the cellularization of a category of modules over the underlying diagram of rings. We explain here that the second step in that zig-zag can be constructed as a combination of the left and right adjoint cases discussed above.

As in Section 4, assume we are given a finite, inverse category $\mathbf{D}$ with at most one morphism in each $\mathbf{D}(s, t)$ and a diagram of ring spectra, $R$, indexed on $\mathbf{D}$. Note that a finite, inverse category is also a direct category. Let $\mathbf{D}_{+}$be the category $\mathbf{D}$ with one added object $z$ and one morphism from $z$ to each object in $\mathbf{D}$ so that $z$ is an initial object in $\mathbf{D}_{+}$. Let + denote the category with one object and one morphism. We next consider the right and left adjunction theorems above applied to the inclusions of $\mathbf{D}$ and + into $\mathbf{D}_{+}$.

Let $f R$ be the fibrant replacement of $R$ and let $\hat{R}$ denote its inverse limit as in Section 4. We extend the diagram $f R$ on $\mathbf{D}$ to the diagram $f R_{+}$on $\mathbf{D}_{+}$such that $f R_{+}(z)=\hat{R}$. By Theorem [5.5 inclusion $i: \mathbf{D} \rightarrow \mathbf{D}_{+}$induces a Quillen adjunction $\left(i^{*}, i_{!}\right)$on the diagram-injective model structures between $f R_{+}$and $f R$ modules. Consider the diagram $f R$ in $f R$-modules and its image $\underline{i}_{!} f R$ in $f R_{+-}$ modules. Since $f R$ is fibrant, $\underline{\underline{i}}_{!} f R(z)=\hat{R}$ and $\underline{\underline{i}}_{!} f R=f R_{+}$. So Theorem [5.5(2) implies the following.

Corollary 5.6. The adjoint functors $\left(i^{*}, i_{!}\right)$induce a Quillen equivalence on the associated cellularizations,

$$
f R_{+}-\text {cell- } f R_{+}-\bmod \simeq_{Q} f R \text {-cell- } f R \text {-mod } .
$$

Proof. The restriction $\underline{\mathrm{i}}^{*} \underline{\mathrm{i}}_{f} f R=\underline{\mathrm{i}}^{*} f R_{+}$is weakly equivalent to $f R$. The same argument that $R$ is small in the proof of Proposition 4.1 also shows that $f R_{+}$and $f R$ are small in $f R_{+}$and $f R$-modules.

Next we consider the inclusion of + in $\mathbf{D}_{+}$with image the object $z$. Again we consider modules over the diagram of rings $f R_{+}$on $\mathbf{D}_{+}$. Restricting to + , $\underline{i}^{*} f R_{+}(z)=\hat{R}$. By Theorem 5.3 inclusion $i:+\rightarrow \mathbf{D}_{+}$induces a Quillen adjunction $\left(i_{*}, i^{*}\right)$ on the diagram-projective model structures between $\hat{R}$ and $f R_{+}$-modules. Next we note that $\underline{\mathrm{i}}_{*} \hat{R}(s)$ is weakly equivalent to $f R(s)$ for any object $s$ in $\mathbf{D}_{+}$. So Theorem 5.3(1) implies the following. 
Corollary 5.7. The adjoint functors $\left(i_{*}, i^{*}\right)$ induce a Quillen equivalence on the associated cellularizations,

$$
\hat{R} \text {-cell- } \hat{R}-\bmod \simeq_{Q} f R_{+} \text {-cell-f } R_{+}-\text {mod } .
$$

As in the end of the proof of Proposition 4.1 we note here that $\hat{R}$ already generates $\hat{R}$-modules so the cellularization on the left is unnecessary in Corollary 5.7. Putting together Corollaries 5.6 and 5.7 and using the Quillen equivalence between the diagram-projective and diagram-injective model structures on $f R_{+}$-cell- $f R_{+}$-mod gives a zig-zag of Quillen equivalences between $\hat{R}$-modules and $f R$-cell- $f R$-modules. As in the proof of Proposition 4.1, Lemma 4.2 and Corollary A.2 then show that $f R$ can be replaced by $R$.

Remark 5.8. One could consider a given general diagram $R$ on $\mathbf{D}$ instead of $f R$ and proceed as in Corollary 5.6. To extend this to a diagram on $\mathbf{D}_{+}$one would need to use the inverse limit $R^{\prime}$ of $R$ at the object $z$ in $\mathbf{D}_{+}$. The derived functor $\underline{i}_{\text {! }}$ evaluated at $z$ applied to $R$ would be $\hat{R}$, the homotopy limit of $R$ or the inverse limit of a fibrant replacement of $R$. Here the limit, $R^{\prime}$, and the homotopy limit, $\hat{R}$, are not weakly equivalent in general. This would cause problems for the steps in Corollary [5.7, because using restriction would force one to work over $R^{\prime}$-modules.

\section{Appendix A. Cellularization of model Categories}

The Quillen equivalences developed in the body of this paper rely on the process of cellularization (also known as right localization or colocalization) of model categories from [12. In [10, we show that Quillen adjunctions between stable model categories induce Quillen equivalences between their respective cellularizations provided there is an equivalence on the chosen cells.

Here we always consider stable cellularizations of stable model categories. We say a set $\mathcal{K}$ is stable if it is closed under suspension and desuspension up to weak equivalence. Given a stable model category $\mathcal{M}$ and $\mathcal{K}$ a stable set of objects, $\mathcal{K}$ cell- $\mathcal{M}$ is again a stable model category by [2,4.6]. We say an object $K$ is small in the homotopy category (simply small elsewhere in the paper) if, for any set of objects $\left\{Y_{\alpha}\right\}$, the natural map $\bigoplus_{\alpha}\left[K, Y_{\alpha}\right] \longrightarrow\left[K, \bigvee_{\alpha} Y_{\alpha}\right]$ is an isomorphism.

Proposition A.1 ([10]). Let $\mathcal{M}$ and $\mathcal{N}$ be stable, cellular, right proper model categories with $F: \mathcal{M} \rightarrow \mathcal{N}$ a left Quillen functor with right adjoint $U$. Denote the associated derived functors by $\underline{F}$ and $\underline{U}$.

(1) Given $\mathcal{K}=\left\{A_{\alpha}\right\}$ a stable set of small objects in $\mathcal{M}$, let $\underline{F \mathcal{K}}=\left\{\underline{F} A_{\alpha}\right\}$ be the corresponding set of objects in $\mathcal{N}$. If for each $A_{\alpha}$ the image $\underline{F} A_{\alpha}$ is small and $A_{\alpha} \rightarrow \underline{U F} A_{\alpha}$ is a weak equivalence, then the $\mathcal{K}$-cellularization of $\mathcal{M}$ and the $\underline{F} \mathcal{K}$-cellularization of $\mathcal{N}$ are Quillen equivalent:

$$
\mathcal{K}-\text { cell- } \mathcal{M} \simeq_{Q} \underline{F} \mathcal{K}-\text { cell- } \mathcal{N} .
$$

(2) Given $\mathcal{L}=\left\{B_{\beta}\right\}$ a stable set of small objects in $\mathcal{N}$, let $\underline{U} \mathcal{L}=\left\{\underline{U} B_{\beta}\right\}$ be the corresponding set of objects in $\mathcal{N}$. If for each $B_{\beta}$ the image $\underline{U} B_{\beta}$ is small and $\underline{F U} B_{\beta} \rightarrow B_{\beta}$ is a weak equivalence, then the $\mathcal{L}$-cellularization of $\mathcal{N}$ and the $\underline{U} \mathcal{L}$-cellularization of $\mathcal{M}$ are Quillen equivalent:

$$
\underline{U} \mathcal{L} \text {-cell- } \mathcal{M} \simeq_{Q} \mathcal{L} \text {-cell- } \mathcal{N} .
$$


If $F$ and $U$ induce a Quillen equivalence on the original categories, then the hypotheses in Proposition A.1 are automatically satisfied. Thus, the cellularizations are Quillen equivalent.

Corollary A.2 ([10]). Let $\mathcal{M}$ and $\mathcal{N}$ be stable, cellular, right proper model categories with $F: \mathcal{M} \rightarrow \mathcal{N}$ a Quillen equivalence with right adjoint $U$. Denote the associated derived functors by $\underline{F}$ and $\underline{U}$.

(1) Given $\mathcal{K}=\left\{A_{\alpha}\right\}$ a stable set of small objects in $\mathcal{M}$, let $\underline{F} \mathcal{K}=\left\{\underline{F} A_{\alpha}\right\}$ be the corresponding set of objects in $\mathcal{N}$. Then the $\mathcal{K}$-cellularization of $\mathcal{M}$ and the $\underline{F} \mathcal{K}$-cellularization of $\mathcal{N}$ are Quillen equivalent:

$$
\mathcal{K}-\text { cell- } \mathcal{M} \simeq_{Q} \underline{F} \mathcal{K}-\text { cell- } \mathcal{N} .
$$

(2) Given $\mathcal{L}=\left\{B_{\beta}\right\}$ a stable set of small objects in $\mathcal{N}$, let $\underline{U} \mathcal{L}=\left\{\underline{U} B_{\beta}\right\}$ be the corresponding set of objects in $\mathcal{N}$. Then the $\mathcal{L}$-cellularization of $\mathcal{N}$ and the $\underline{U} \mathcal{L}$-cellularization of $\mathcal{M}$ are Quillen equivalent:

$$
\underline{U} \mathcal{L} \text {-cell- } \mathcal{M} \simeq_{Q} \mathcal{L} \text {-cell- } \mathcal{N} .
$$

This corollary also follows from the dual of [15, 2.3].

\section{REFERENCES}

[1] Vigleik Angeltveit, Enriched Reedy categories, Proc. Amer. Math. Soc. 136 (2008), no. 7, 2323-2332, DOI 10.1090/S0002-9939-08-09185-5. MR2390498(2009a:18009)

[2] David Barnes and Constanze Roitzheim, Stable left and right Bousfield localisations, Glasg. Math. J. 56 (2014), no. 1, 13-42, DOI 10.1017/S0017089512000882. MR.3137847

[3] Clark Barwick, On left and right model categories and left and right Bousfield localizations, Homology, Homotopy Appl. 12 (2010), no. 2, 245-320. MR.2771591 (2012a:18031)

[4] Tibor Beke, Sheafifiable homotopy model categories, Math. Proc. Cambridge Philos. Soc. 129 (2000), no. 3, 447-475, DOI 10.1017/S0305004100004722. MR.1780498(2001i:18015)

[5] Julia E. Bergner, Homotopy fiber products of homotopy theories, Israel J. Math. 185 (2011), 389-411, DOI 10.1007/s11856-011-0116-3. MR2837143(2012i:18016)

[6] Julia E. Bergner, Homotopy limits of model categories and more general homotopy theories, Bull. Lond. Math. Soc. 44 (2012), no. 2, 311-322, DOI 10.1112/blms/bdr095. MR2914609

[7] J. Cirici, Cofibrant models of diagrams: mixed Hodge structures in rational homotopy, to appear in Trans. Amer. Math. Soc., arXiv: 1307.4968

[8] J. P. C. Greenlees and B. Shipley, An algebraic model for free rational G-spectra for connected compact Lie groups G, Math. Z. 269 (2011), no. 1-2, 373-400, DOI 10.1007/s00209-010-07412. MR2836075 (2012h:55012)

[9] J. P. C. Greenlees and B. E. Shipley, An algebraic model for rational torus-equivariant stable homotopy theory, Reorganized version in preparation.

[10] J. P. C. Greenlees and B. Shipley, The cellularization principle for Quillen adjunctions, Homology Homotopy Appl. 15 (2013), no. 2, 173-184, DOI 10.4310/HHA.2013.v15.n2.a11. MR 3138375

[11] Kathryn Hess and Brooke Shipley, The homotopy theory of coalgebras over a comonad, Proc. Lond. Math. Soc. (3) 108 (2014), no. 2, 484-516. MR3166360

[12] Philip S. Hirschhorn, Model categories and their localizations, Mathematical Surveys and Monographs, vol. 99, American Mathematical Society, Providence, RI, 2003. MR.1944041 (2003j:18018)

[13] A. Hirschowitz and C. Simpson, Descente pour les $n$-champs (Descent for $n$-stacks), arXiv: math/9807049.

[14] Mark Hovey, Model categories, Mathematical Surveys and Monographs, vol. 63, American Mathematical Society, Providence, RI, 1999. MR.1650134 (99h:55031)

[15] Mark Hovey, Spectra and symmetric spectra in general model categories, J. Pure Appl. Algebra 165 (2001), no. 1, 63-127, DOI 10.1016/S0022-4049(00)00172-9. MR.1860878 (2002j:55006)

[16] T. Huettemann and O. Roendigs, Twisted diagrams and homotopy sheaves, arXiv:0805.4076. 
[17] Mark W. Johnson, On modified Reedy and modified projective model structures, Theory Appl. Categ. 24 (2010), no. 8, 179-208. MR2720182(2011i:55026)

[18] Stefan Schwede and Brooke Shipley, Stable model categories are categories of modules, Topology 42 (2003), no. 1, 103-153, DOI 10.1016/S0040-9383(02)00006-X. MR1928647 (2003g:55034)

[19] Bertrand Toën, The homotopy theory of dg-categories and derived Morita theory, Invent. Math. 167 (2007), no. 3, 615-667, DOI 10.1007/s00222-006-0025-y. MR2276263 (2008a:18006)

Department of Pure Mathematics, The Hicks Bullding, University of Sheffield, ShefFIELD, S3 7RH, UNITED KINGDOM

E-mail address: j.greenlees@sheffield.ac.uk

Department of Mathematics, Statistics and Computer Science, University of Illinois at Chicago, 508 SEO m/C 249, 851 S. Morgan Street, Chicago, Illinois 60607-7045

E-mail address: bshipley@math.uic.edu 